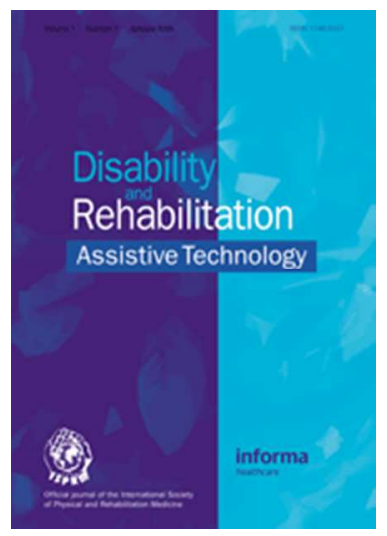

\title{
An investigation of factors affecting the performance of wheelchair transfers
}

\begin{tabular}{|r|l|}
\hline Journal: & Disability and Rehabilitation: Assistive Technology \\
\hline Manuscript ID & TIDT-11-2017-001.R1 \\
\hline Manuscript Type: & Original Research \\
\hline Keywords: & Assistive Technology, Survey, Wheelchair Transfers, Upper Limb Pain \\
\hline \multicolumn{2}{|l}{} \\
\hline
\end{tabular}

\section{SCHOLARONE ${ }^{m}$}

Manuscripts 


\title{
An investigation of factors affecting the performance of wheelchair transfers
}

\author{
Purpose: Being able to transfer in and out of their wheelchair is an important task \\ for wheelchair users that can be affected by a variety of different factors. The aim \\ of this study was to investigate the interplay between personal characteristics, \\ wheelchair users' transferring technique and the other factors that can affect the \\ performance of wheelchair transfers.
}

Method: A cross-sectional survey study was designed to recruit wheelchair users, aged 18 or older, performing sitting-pivot or standing-pivot wheelchair transfers, independently or with assistance.

Results: 42 usable surveys were returned. Transferring technique is usually determined by the individual's impairment. In turn, transferring technique will greatly affect the perceived difficulty of different types of wheelchair transfers and, the number of transfers that people will perform in a day. The presence of upper limb pain increased the difficulty reported for certain types of transfers while the use of assistive technologies appears to be only partially successful in facilitating the performance of wheelchair transfers.

Conclusion: This study highlights the complexity of the factors included in the performance of wheelchair transfers. Findings from this survey can be utilised to broaden the boundaries of future research.

Keywords: wheelchair transfers; survey; assistive technologies; upper limb pain

\section{Introduction}

Wheelchair transfers are one of the most important activities in the lives of wheelchair users $[1,2]$. Wheelchair users represent an extremely broad and diverse group that includes people with different medical conditions, demographic characteristics, lifestyles, needs, difficulties and goals [3]. Depending on their individual characteristics, some people will be able to perform wheelchair transfers independently, while others might need partial or complete assistance. Furthermore, some people who 
transfer independently might adopt a standing-pivot or a sitting-pivot technique while others might rely on assistive technologies (ATs) to facilitate their performance [4]. Due to this diversity, wheelchair users will have different needs and employ different strategies when transferring to and from their wheelchair. In addition transfers take place in a different settings.

Previous studies have estimated that, on average, a person with spinal cord injury (SCI) transfers to and from their wheelchair approximately 15 times a day [5-7]. However, the number of transfers reported by each individual can vary from 0 to 45 transfers per day [5]. To our knowledge, no study so far has investigated the causes of such a large variation.

Several factors can affect the individual's ability to perform wheelchair transfers. Studies by Dalyan et al [8] and Samuelsson et al [9] reported that presence and the intensity of upper limb pain interfered with the performance of wheelchair transfers for $74 \%$ and $62 \%$ wheelchair users with SCI. Transferring, particularly transferring into and out of a car, is one of the items on the Wheelchair Users Shoulder Pain Index (WUSPI) which receives the highest reported intensity of pain by wheelchair users [10]. However, these studies give us no indication of how pain impacts the performance of wheelchair transfers; nor if and how people with different impairments are affected by the presence of pain during transfers.

Several studies have also identified how different types of wheelchair transfers (wheelchair-toilet, wheelchair-car, wheelchair-bed) can be more or less difficult for the individual and potentially lead to increased physical effort $[11,12]$. Additionally, Toro et al [13] measured the influence of different environmental factors such as height difference, gap dimensions, the presence of an obstacle, the presence and height of a frontal or lateral grab bar on the performance of wheelchair transfers. Results indicated 
how height difference between wheelchair and target seat, both higher and lower, and the presence of an obstacle had the greatest impact on the participant's ability to transfer. Placing a grab bar in front of the target seat helped approximately $20 \%$ of the individuals during several of the transfers performed. Although results from these studies might be helpful to understand objective difficulties that wheelchair users in general encounter when transferring in the built environment, they fail to consider how these difficulties might vary across participants using different transferring techniques. Few studies have taken into account the use of Assistive Technologies (ATs) in the evaluation of wheelchair transfers [14] and none of them have investigated the likelihood of transfer's ATs use among people with various levels of independence nor how the use of ATs impacts the perceived difficulties of wheelchair transfers. Finally, psychological factors such as confidence and self-efficacy have also been demonstrated to have an important effect on performance level during wheelchair sports [15].

Furthermore, studies from Best et al [16] and Sakakibara et al [17] have shown how increased confidence and self-efficacy of manual wheelchair skills results in improved performance that positively affect participation. Wheelchair transfers are described as an important and challenging activity for many wheelchair users [1,11] but how does the level of independence and the technique used by the individual affects selfconfidence and satisfaction?

Most studies on wheelchair transfers have focussed on one aspect related to the performance of wheelchair transfers such evaluating the impact of upper limb pain [10] or transfer set up and environmental constraints [13]. Moreover, the majority of available studies eithers focuses on the performance of independent sitting transfers performed by people with SCI [18] or generalises findings across many wheelchair 
users regardless of their level of independence and the technique used when transferring.

This study has a two-fold aim. The first is to identify general characteristics of wheelchair users who perform wheelchair transfers with various levels of independence and using different transferring techniques. The second aim is to explore which factors affecting the performance of wheelchair transfers are dependent on the transferring technique adopted by the individual and which factors are relevant regardless of how the transfer itself is performed.

\section{Method}

\section{Materials and Procedure}

A five section cross-sectional self-administered survey was purposefully designed by the authors for the study. The questionnaire was developed based on a literature review concerning factors that can potentially influence the performance of wheelchair transfers, such weight, type of impairment, transferring technique, need for assistance, $\underline{\text { use of ATs and context of the transfer [13,19-22]. Additionally, as it was hypothesised }}$ $\underline{\text { that perceived competency and self-efficacy in might directly affect the performance of }}$ wheelchair transfers, four questions from the Intrinsic Motivation Inventory [23] were included in the questionnaire. A multiple choice response format was developed for the majority of questions to reduce time and burden of participation $[24][23]$. These questions required participants to use a 7 point scale to report their response. To maintain consistency, as the Intrinsic Motivation Inventory [23] features rating questions using a 7 point Likert scale, all rating question throughout the survey used the same format. At the end of the questionnaire an open ended question was added, asking for additional comments, insights or feedback from respondents. The survey was 


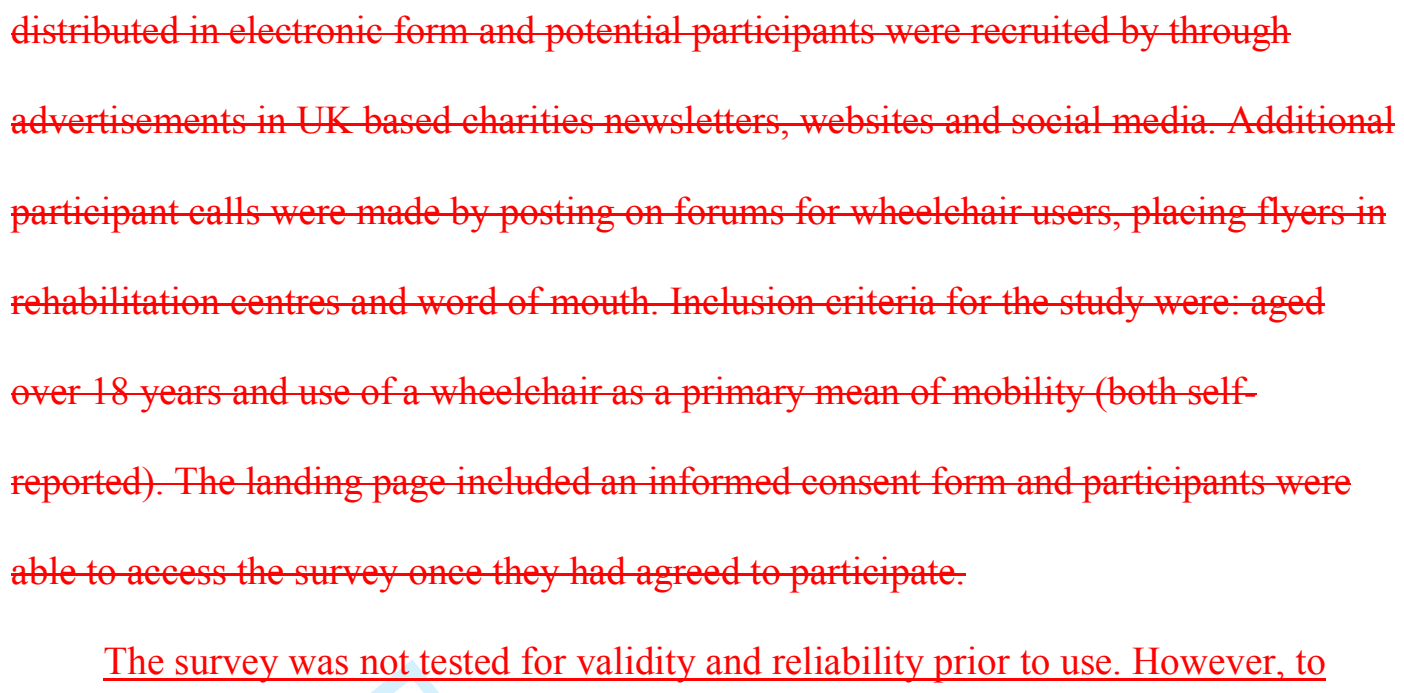

(1) Demographic Information (8 Questions): General characteristics of the respondent were collected in this section including the subject's gender, age, weight, height, and the experience as a wheelchair users (in months and years), the primary medical condition, the primary type of wheelchair used and any additional wheelchairs used.

(2) Wheelchair Transfer Characteristics (5 Questions): Questions in this section explored general characteristics of wheelchair transfers normally performed by the respondent such as the need for assistance during transfers, the use of a sitting-pivot vs standing-pivot technique, the number of transfers performed on a daily basis, the type of transfers normally performed (bed, bathroom, or other 
transfers in the house, car and also transfer between wheelchairs) and the perceived difficulty for type of transfers performed.

(3) Use of Assistive TechnologiesATs (6 Questions): Items in this section were divided into two parts. In the first part, respondents were asked to report the use of different ATs for wheelchair transfers (transfer boards, hoists, grab bars, fitted modifications such as swivel seats or other ATs) and to categorise their use according to the different types of transfers normally performed. The first, assessed the use of various ATs categorized by the type of wheelchair transfer performed. The second part, captured an overview of the main advantages and difficulties of using ATs, the self-reported ease of use for each reported AT and motivations for non-use.

(4) Presence and Intensity of Pain (3 Questions): This section consisted of two items aimed at assessing the presence-location (shoulder, elbow, wrist, hand or other) and intensity of upper limb recurring pain within the last six months.

Additionally, a third question was added to explore the frequency of pain exacerbated by wheelchair transfers.

(5) Wheelchair Transfer Motivation (1 Question): This section evaluated four sections of the Intrinsic Motivation Inventory [23][24], namely: importance attributed to the taskskill level, the effort invested in the performance, the perceived competence of the subject and the pressure related to the activity execution.

The survey was distributed in electronic form and potential participants were recruited by through advertisements in UK based charities newsletters, websites and social media. Additional participant calls were made by posting on forums for wheelchair users, placing flyers in rehabilitation centres and word of mouth. Inclusion criteria for the 
study were: aged over 18 years and use of a wheelchair as a primary mean of mobility

(both self-reported). The landing page included an informed consent form and

participants were able to access the survey once they had agreed to participate.

\section{Data Analysis}

Data from completed surveys were analysed using IBM SPSS Statistics Version 22 (IBM Corporation, NY, USA). Descriptive statistics were calculated in order to illustrate demographic and general characteristics of the respondents, types of transfers performed, advantages and disadvantages of ATs. Respondents were divided in 3 primary groups according to the level of assistance needed when transferring and their transferring technique (independent sitting transfers, independent standing transfers, assisted transfers). Associations between wheelchair users' demographic characteristics and transferring technique and level of assistance were explored using cross tabulation and a Chi-squared test. A one-way analysis of variance (ANOVA) was used to determine the impact of assistance and technique on the frequency of wheelchair transfers performed on a daily basis.Data related to the daily frequency of transfers was checked for normality using the Saphiro Wilk test. Due to the non-normality of the data across different groups a Kruskal-Wallis test was used to determine the impact of $\underline{\text { technique and assistance on the frequency of wheelchair transfers performed on a daily }}$ basis. A chi-squared test was employed to investigate the association between participants' technique, presence of pain and AT use. A Kruskal-Walllis test was used to analyse the impact of transfer technique and independence on intensity of pain, reported difficulty, individual's motivation and self-satisfaction. Where significant differences were found Tukey test was used for ANOVA post hoc analysis and Dunn's test with Bonferroni correction for Kruskal-Wallis pairwise comparison, while standardised residuals were examined for Chi-squared tests. Statistical tests were chosen 
according to the type of independent variable: ANOVA for continuous variables, Kruskal-Walllis test for ordinal variables and chi-squared test for nominal variables. Spearman's correlation was used to assess the relationship between satisfaction with transfer performance and reported difficulty across different transferring techniques. Lastly, independent t-test were employed to assess the impact of participant's weight on the likelihood of AT use during transfers. Level of significance for all statistical tests was set at .05 .

\section{Results}

\section{Participants}

A total of 42 fully completed surveys were returned. Demographic characteristics are presented in Table 1. The median age of the respondents was 46.5 years, ranging from 19 to $67(\mathrm{IQR}=22)$. Median self-reported weight and height were, respectively $72.3 \mathrm{~kg}$ and $172.7 \mathrm{~cm}$, ranges spanned between $30.4 \mathrm{~kg}$ to $120 \mathrm{~kg}(\mathrm{IQR}=33.3)$ and $122 \mathrm{~cm}$ to $195.6 \mathrm{~cm}(\mathrm{IQR}=21)$. Respondents were on average very experienced wheelchair users, nearly $55 \%$ had more than 10 years of experience. Median number of years of experience as a wheelchair users was 13.25 . Primary medical conditions varied greatly across respondents. The most common reported diagnosis was SCI, followed by MS and cerebral palsy (CP). However, over $35 \%$ of the respondents had other medical conditions which affected their mobility including Ehlers-Danlos Syndrome, muscular dystrophy and post-polio paralysis. Respondents were mainly manual wheelchair users, with only $30 \%$ reporting powered or pushrim activated power assisted wheelchairs (PAPAW) as a primary mean of mobility. Twenty-seven respondents also reported the ownership and use of other wheelchairs and mobility devices, including scooters, sport wheelchairs and add-on hand bikes. From cross tabulation of demographic factors the 
sample appears very homogeneous, with even distribution of age groups and physical characteristics across different medical conditions and primary wheelchair type. More clear relationships were instead found between medical condition and type of primary wheelchair used, with the totality of respondent who reported a low level SCI using rigid and foldable frame wheelchairs as a primary mean of mobility.

\section{$\underline{\text { Table } 1}$}

\section{Wheelchair users characteristics according to level of independence and transfer strategies}

Thirty-one (73.8\%) of the respondents reported being able to transfer without any assistance. Of these $23(74.2 \%)$ performed sitting-pivot transfers while 8 transferred using a standing-pivot technique. Seven participants (16.7\%) stated that they usually need some assistance in order to safely transfer in and out of their wheelchair and 6 (85.7\%) used a sitting-pivot technique. Only 4 respondents, accounting for $9.5 \%$ of the total population, defined themselves as completely dependent when performing wheelchair transfers and no one transferred using a sitting-pivot technique. Due to the low number of participants performing assisted and dependents transfers, their responses were combined while independent wheelchair transfers were categorised according to the technique (sitting-pivot or standing-pivot) used by the individual. A significant relationship was found between individuals' medical condition and transferring strategy $\chi^{2}=32.43$, and $p<0.001$. Over $60 \%$ of the respondents performing independent sitting-pivot wheelchair transfers reported having a SCI that only affected their lower limbs, while $74.1 \%$ of the tetraplegic participants stated that they needed partial or complete assistance when performing transfers. All participants with $\mathrm{CP}$ performed independent standing-pivot transfers while respondents with other, various medical conditions were more evenly spread across the 3 transferring techniques. No 
significant association was found between participants' transferring technique and primary type of wheelchair used $(\mathrm{p}=.07)$ or individuals' body weight $(\mathrm{p}=.316)$.

Figures 1, 2 and 3 show the distribution of participants' characteristics across the 3 transferring techniques.

\section{Figure 1}

\section{Figure 2}

\section{Figure 3}

\section{Frequency of transfers, types of transfers and reported difficulty}

The median number of transfers performed daily by respondents was $8(\mathrm{IQR}=5.3)$.

There was a statistically significant difference between transferring technique groups as determined by the Kruskal Wallis Test $(\chi 2(2)=8.128, \mathrm{p}=.017)$. A Dunn's post-hoc test with Bonferroni correction revealed that the daily frequency of transfers was

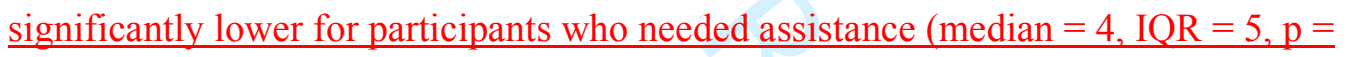
.016) compared to participants who performed independent sitting transfers (median $=$ $\underline{8, I Q R}=9)$. The median number of daily transfers reported by participants performing independent standing transfers was $7.5(\mathrm{IQR}=3.5)$ but the difference was not significant when compared to the other 2 groups (lowest $p=.37$ ). Participants performed on average 9 wheelchair transfers per day $(\mathrm{STD} \pm 7)$. The number of transfers per day was significantly difference between transferring technique groups as determined by ene-way ANOVA $(\mathrm{F}(2,39)=4.088, \mathrm{p}=.024)$. A Tukey post hoc test revealed that the daily frequency of transfers was significantly lower for participants who needed assistance $(4.6 \pm 2.4 \mathrm{~min}, \mathrm{p}=.022)$ compared to participants who performed independent sitting pivot transfers $(11.6 \pm 8.8 \mathrm{~min})$. Participants performing independent standing pivot transfers reported an average of $7.6 \pm 2.5$ transfers per day but the difference was not significant when compared to the other 2 groups (lowest $p=$ 
When asked about the different types of transfers routinely performed, all respondents reported performing bed to wheelchair transfers and various transfers from

everyday base. Car transfers were also very common, with $76.2 \%$ of respondents performing them daily, other house transfers (e.g.: couch, dining table) were slightly less common $64.3 \%$, while transfers between different wheelchairs were a regular occurrence for $69 \%$ of the respondents. Additionally, thirteen participants reported frequent transfers onto office chairs, restaurants and/or cinema seats, plane seats and various exercise or rehabilitative devices such as hand bikes and standing frames. When looking at the types of transfers performed by individuals by transferring technique group, we found that car transfers were performed by $91.3 \%$ of the participants using an independent sitting-pivot transferring technique. Respectively, only $62.5 \%$ and $54.5 \%$ of wheelchair users performing standing-pivot and assisted transfers reported regular transfers to and from car seats. Similarly, people using an independent sitting-pivot technique were more likely to perform house transfers (69.6\%) compared to individuals performing independent standing-pivot transfers $(62.5 \%)$ or assisted transfers $(54.5 \%)$. Transfers between wheelchairs were common practice for all respondents using an independent standing-pivot technique while they were performed by only $65.2 \%$ of individuals using independent sitting-pivot technique and $54.5 \%$ of people performing assisted transfers.

Participants were also asked to rank the level of difficulty of each transfer they routinely perform on a Likert scale from 1 (Very Easy) to 7 (Very Hard). When considering all participants within a single group, bed transfers were considered the easiest among the more frequent transfers (Mean 2.74, $\mathrm{STD} \pm 1.71$ ). House transfers are 
also performed with moderate ease (Mean 3.00, $\mathrm{STD} \pm 1.47$ ), while bathroom transfers, transfers between wheelchairs and car transfers appear as a more challenging scenarios (Means 3.76, 3.79, 3.94, STDs $\pm 1.88,1.92,1.95$ ). People who reported additional routine execution of other transfer types, in addition to the five basic scenarios we proposed (bed, bathroom, car, house and between wheelchairs), rated them as generally easy (Mean 2.62, STD \pm 1.39 ). When responses were examined separately according to participants' transferring technique, it was observed that reported average difficulties were lower for individuals performing independent sitting transfers across all conditions. Kruskal Wallis Test shows a statistically significant difference between groups for bed $\left(\chi^{2}(2)=10.317, p=.006\right)$, $\operatorname{car}\left(\chi^{2}(2)=2.564, p=.020\right)$, house $\left(\chi^{2}(2)=\right.$ $12.03, p=.002)$ and other transfers $\left(\chi^{2}(2)=7.5, p=.024\right)$. Post hoc analysis revealed how all transfers were considered significantly easier by participants using an independent sitting-pivot technique compared to individuals who required assistance (bed transfers $\mathrm{p}=.009$, car transfers $\mathrm{p}=.016$, house transfers $\mathrm{p}=.002$, other transfers $\mathrm{p}$ $=.041)$. Figure 4 shows the mean difficulty reported for different types of transfers by respondents using different transferring techniques.

\section{$\underline{\text { Figure } 4}$}

\section{Presence and influence of pain on transfer performance}

As expected, painful symptomatology in one or more areas of the upper limbs was reported by the majority of the respondents. Complaints of shoulder and wrist pain in the previous six months were the most common, affecting respectively $69 \%$ and $52.4 \%$ of the respondents. Frequencies of hand and elbow discomfort were only slightly lower, respectively $50 \%$ and $42.9 \%$. Additionally, $52.4 \%$ of participants reported recurring pain in other body locations such as neck, back and buttocks. The relationship between upper limb pain and wheelchair transfer was confirmed by the fact that $66.6 \%$ of the 
respondents complained that their pain was exacerbated by wheelchair transfers at least occasionally. Average intensity of pain, was moderately high for all upper limb's districts pain with values ranging from 4 at the elbow to 4.45 at the wrist. Chi-squared test revealed no significant association between presence of pain during transfers, and the technique used for transferring $(p=.236)$. Furthermore, Kruskal Wallis Test confirmed that there was no significant relationship between the wheelchair users' transferring technique and the intensity of shoulder $(p=.311)$, elbow $(p=.680)$, wrist $(\mathrm{p}=.550)$ and hand $(\mathrm{p}=.974)$ pain. The presence of upper limb pain during transfers did not significantly affected the number of daily transfers for individuals performing independent sitting $(p=.765)$, independent standing $(p=.656)$ or assisted transfers $(\mathrm{p}=.082)$. Overall, participants who complained of frequent upper limbs pain during transfer performance generally reported higher level of difficulty for all transfer types. However, this difference was deemed significant only for bed $\left(\chi^{2}(3)=8.417, p=\right.$ $.038)$ and house transfers $\left(\chi^{2}(3)=9.023, p=.029\right)$. Post-hoc analysis showed that in both cases the differences were only significant when comparing respondents whose pain is always exacerbated by transfers and the other three groups (highest significant $p$ $=.043)$.

\section{Motivation and satisfaction with transfer performance}

Over $88 \%$ of respondents considered transferring skills extremely important, giving the maximum score of 7 on the provided Likert scale. This was further confirmed by the fact that, when asked about the effort invested in the task, $85.7 \%$ of the respondents stated that they invested great effort in performing wheelchair transfers safely and effectively (Score 6 and 7 on Likert scale). Participants were generally, satisfied with the way they transferred in and out of their wheelchair and they felt reasonably secure during the performance. However, $16.7 \%$ of respondents were unsatisfied with their 
skills (1-3 points on Likert scale) and 28.6\% felt extremely tense during wheelchair transfers (Score 6 and 7 on Likert scale). Transferring technique and need for assistance did not seem to impact the importance people attributed to wheelchair transfers $(\mathrm{p}=$ $.137)$, the satisfaction with their ability $(\mathrm{p}=.17)$ nor the perceived stress during wheelchair transfers $(p=.119)$. However, a significant relationship was found between the transferring technique used by the participant and the effort invested in performing safe transfers $\left(\chi^{2}(2)=7.575, p=.023\right)$. Dunn's test showed that participants performing independent sitting-pivot transfers tried harder to perform safe transfers $(6.65 \pm 1.08)$ compared to participants using an independent standing-pivot technique $(5.75 \pm 1.39$ $\min , \mathrm{p}=.019)$ and participants performing assisted transfers $(5.73 \pm 1.95 \mathrm{~min}, \mathrm{p}=.039)$. Regardless of their transferring technique, participants who reported increased difficulties for various types of transfers were less likely to be satisfied with their transferring skills $(r=-.505, p<.001)$. Furthermore, pain during transfer also had an effect on how nervous respondents felt during transfer performance $\left(\chi^{2}(3)=9.788, \mathrm{p}=\right.$ .020). Post hoc analysis revealed how differences in the level of perceived insecurity are significant only for respondents complaining of occasional pain during transfers $(2.95 \pm$ $2.16 \mathrm{~min}, \mathrm{p}=.012)$ and respondents reporting constant pain during transfers $(5.88 \pm$ $1.25)$.

\section{Use and needs for ATs}

The vast majority of respondents, $73.8 \%$, declared to benefit, in one or more situation, from the use of an $\underline{\mathrm{AT}}$ assistive technology or from the presence of environmental modifications such as swivel seats or adapted vehicles. Overall, grab bars were the most commonly adopted, used by $42.9 \%$ of participants, followed by transfer board, hoists, environmental modifications and other less common devices. . However, the type of transfer for which each assistive technology $\underline{\mathrm{AT}}$ is usually employed differ from one 
device to the other. Grab bars were found almost exclusively within the house, particularly in the bathroom. Only four individuals declared having special handles mounted in their car in order to ease their transfers. On the other hand, the use of transfer boards was slightly more common for car transfers $(33.3 \%)$, compared to bed $(23.3 \%)$ or other house transfers $(20 \%)$. As expected, hoists were found only within the house and used mainly for bathroom, bed, house or transfers between different wheelchairs. Reported environmental modifications concerned adapted vehicles in $66.7 \%$ of cases and hospital beds in the remaining $33.3 \%$.

We found no significant relationship between the likelihood of AT use and the transferring technique adopted by the subject $(p=.28)$. Surprisingly, the choice of AT was only mildly affected by the individual's transferring technique. Transfer boards were more common among participants performing independent sitting-pivot transfers, while grab bars used more by participants who transferred while standing up. However, the difference in AT distribution was only significant for hoists which were used almost exclusively by people needing assistance for transferring $(p=.025)$. Regardless of participant's transferring technique, no association was found between the presence of pain during transfers and the use of ATs. However, as shown in Figure 5, participants who reported use of various ATs during transfers were found to be significantly heavier (78.603 \pm 22.186$)$ than participants who transferred without using any AT (63.764 \pm 16.256) $\mathrm{t}(40)=-2.027, \mathrm{p}=.049$.

\section{Figure 5}

\section{Discussion}

Most studies on wheelchair transfers have focussed their efforts towards attempting to isolate and explain individual variables that affect the performance of wheelchair transfers such as the presence of upper limb pain [10], the configuration of the transfer 
set-up [13] or the impact of specific aspects of the physical impairment affecting the individual [25]. As noted by Crytzer et al [21] this approach is not sufficient to capture the complexity of aspects that can affect the ability of an individual to perform wheelchair transfers. To our knowledge, this is the first study attempting to take a more comprehensive look at the various factors that can affect the performance of wheelchair transfers and explore the potential relationships among them.

Barbareschi et al [19] show how the use of different transferring techniques can have a great impact on the safety of the transfer and the mechanical loading on the upper limbs. As transferring technique plays such an important role in the performance of wheelchair transfers, the first aim of this study was to analyse the individual characteristics that are associated with the performance of sitting-pivot, standing-pivot and assisted transfers. Results from our survey showed that primary medical condition is the main factor that determines the movement strategy adopted by the person when transferring. As weight has been shown to significantly decrease the functional abilities of many wheelchair users [26], we expected individuals with higher bodyweight to be more likely to require assistance during transfers. However, our findings show that, while grater bodyweight increased the likelihood of ATs use, it had no impact on the transferring technique used by participants nor it affected their need for assistance. Results from the survey confirmed the fact that transferring technique had an important impact on the performance of wheelchair transfers in real life. However, we identified several aspects that were crucial for all wheelchair users, regardless of the way in which they transferred.

In the survey carried out by Fliess-Douer et al [1], wheelchair users with different impairments, lifestyles and abilities agreed in rating transfers as the most essential skills in everyday life. Our study shows similar results with $88 \%$ of 
respondents attributing crucial importance to their ability to transfer. Despite the fact that the importance attributed to the task was consistent across all wheelchair users, we found that individuals performing independent sitting wheelchair transfers reported investing more effort in ensuring the safety of their transfers. Wheelchair users who need assistance during transfers might be more likely to rely on their caregivers to make sure that transfers are performed in a safe and efficient manner. On the other hand, we believe that the scarcer safety consideration by wheelchair users performing standing transfers might be related to their lack of awareness. Most wheelchair users are likely to learn about the importance of using efficient and safe technique when transferring during rehabilitative training. Wheelchair skills, and transfers, training are considered an important part of rehabilitation for individuals with SCI [27], who represented a substantial portion of the participants performing independent sitting-pivot transfers. Respondents performing independent standing-pivot transfers were individuals with CP, MS and EDS which makes them less likely to have received comprehensive wheelchair transfers training. Without proper training they might have limited knowledge of how to best perform safe and efficient transfers and be less aware of the potential risks associated.

Reported perceived difficulty of different transfer type was mostly in line with results presented by Janssen et al [12], who measured physical strain, based on individual's hart rate, of several ADL including various wheelchair transfers. Transfers to the car seat and to a shower wheelchair were identified as more strenuous activities, while toilet and bed transfers appeared to be less demanding. In our study, participants identified car transfers and transfers between wheelchairs as more difficult to perform, however bed transfers were considered significantly easier than bathroom transfers. Differences could be due to different experimental set ups, as Janssen et al [12] asked 
participants to perform transfers in a series of standardized environment while we asked people to evaluate the transfers they perform in their own home. Additionally, Janssen et al [12] evaluated three bathroom transfers (toilet, shower bench and shower chair) separately, while in our study they were grouped together as bathroom transfers which might have been confusing for respondents. Transferring technique was found to have an important impact on the perceived difficulty of wheelchair transfers. Unsurprisingly, participants who needed assistance for transferring reported consistently higher scores for transfer difficulty across different transfer types. On the other hand, we expected participants who were able to perform independent standing transfers to report lower difficulties than participants who relied on their upper limbs for transferring. However, even if they are able to reach a standing position, many wheelchair users will lack both lower limbs' strength and balance. This is likely to make their transfers more challenging, despite the fact that their physical impairment appears to be less severe compared to people who need to fully rely on their upper limbs for transferring.

Overall, the average number of transfers performed daily by our respondents was lower than reported in literature. However, in the study carried out by Curtis \& Black [5] and Finley et al [6] included only paraplegic respondents able to perform independent wheelchair transfers. Unsurprisingly, participants who required assistance reported the lowest number of transfers per day. The combination of increased difficulty and their need to rely, at least partially, on caregivers' help, made them more likely to only perform transfers that were essential to them. Similarly, we hypothesize that the higher difficulty reported by individuals performing standing transfers is at least partially responsible of the reduced number of transfers carried out in a day.

The incidence of upper limb pain among our respondents was consistent with findings from literature and appeared unaffected by participants' transferring technique 
$[28,29]$. The interference of upper limb pain during transfer performance identified by Samuelsson et al [9] was confirmed by the fact that over $66 \%$ of participants reported that pain was often exacerbated by the performance of wheelchair transfers. On the other hand, as noted by Finley et al [6] the presence of upper limb pain did not have any significant effect on the number of wheelchair transfers performed in a day. As previously highlighted, the ability to transfer in and out of their wheelchair is crucial for the individual's independence. For this reason, wheelchair users might chose to endure the pain caused by the task in order to not lose their independence. The presence of upper limb pain was also shown to affect the perceived difficulty of transfer performance and increased the sense of anxiety people might experience during the performance of wheelchair transfers. This is particularly important considering transfers have a great influence on the overall fear of falling that wheelchairs users might experience [30]. Anxiety might also negatively affect performance and increase the risk of falls as it has been documented for walking, particularly among elderly respondents [31].

Surprisingly, the choice of using ATs was mostly not affected by the transferring technique of the individual. Additionally, despite the fact that medical guidelines recommend the use of $\underline{\text { ATs }}$ assistive technologies-for wheelchair transfers for all respondents experiencing upper limb pain and/or at risk for upper limb injury [18], we found no relationship between the likelihood of AT use and the presence of upper limb pain during transfers. On the other hand, respondents reporting use of ATs were found to weigh more which suggest that ATs are considered a valuable tool when the task becomes physically strenuous. We also found that several people used more than one AT assistive technology and that the choice can be affected by environmental conditions and the type of transfer to perform. Although this was expected for fitted 
modifications and non-portable devices, such as wheelchair hoists, the use of transfer boards was also found to be subjected to this variability. As illustrated in the study conducted by Haubert et al [20], the environmental conditions in which a wheelchair transfer is performed will have an important effect on the movement strategy of the individual and these changes appear to be relevant regardless of the technique or AT used.

Participants found their ATs assistive technologies-generally easy to use and reported an increased level of confidence when performing transfers. Over $83 \%$ of the respondents reported encountering at least one difficulty when using assistive technologies $\underline{\mathrm{ATS}}$. Frustrations were mainly related to physical limitations of various devices, including excessive weight, bulky profile and instability during transfer performance. Inability to deal with space constraints, low portability and excessive cost were also among the most common reasons participants provided to explain why they chose to not use any

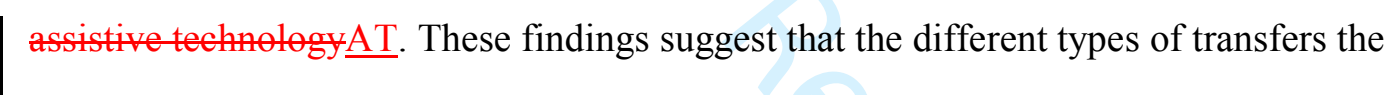
individuals perform and the environmental constraints associated with each type of transfer might affect, beside the transferring technique, also the ability to use

ATsassistive technologies-when needed.

\section{Limitations}

This study provided important new data on the relationship between factors that can affect the performance of wheelchair transfers, however there are limitations that need to be considered. Although our results appear to agree with finding from larger studies, due to the small sample size and the limited age range of respondents' generalization of the results should be carried out with caution. Additionally all our participants where UK residents and their attitude towards several of the investigated factors, including pain, need for assistance and assistive technology AT use might be different from other 
cultures [32]. Lastly, although we invited people to express comment, concern or suggestion concerning additional factors that might affect the performance of wheelchair transfer, the format of an online survey might be too simplistic for such a discussion. Hence we think the complexity of the phenomenon might benefit from additional exploration though qualitative and quantitative studies.

\section{Conclusions}

There are several factors that can affect the performance of transfers in the real world and it is the combination of these individual factors that makes transfers a challenging activity for many wheelchair users.

\section{Figure 6}

FAs shown in Figure 6 transferring technique is usually determined by the individual's impairment. In turn, transferring technique will greatly affect the perceived difficulty of different types of wheelchair transfers and, consequentially the number of transfers that people will perform in a day. On the other hand, the relevance of other factors seems to be unaffected by the transferring technique used by the person. Concerns about the impact of transfers on the development of upper limbs injuries and pain are usually only mentioned for people performing independent sitting-pivot transfers. However, the presence of pain during transfers' performance appears to be a problem concerning all wheelchair users. Currently, AT use was found to be unrelated to both transferring technique and presence of pain during transfers and only linked to increased body weight. Overall, available ATs are only moderately effective in enabling people to perform wheelchair transfer and they can be cause of frustration for the users due to their high cost and limited functionality. 


\section{References}

[1] Fliess-Douer O, Vanlandewijck YC, Van Der Woude LHV. Most Essential Wheeled Mobility Skills for Daily Life: An International Survey Among Paralympic Wheelchair Athletes With Spinal Cord Injury. Arch. Phys. Med. Rehabil. 2012;93:629-635.

[2] Mills T, Holm MB, Trefler E, et al. Development and consumer validation of the Functional Evaluation in a Wheelchair (FEW) instrument. Disabil. Rehabil. 2002;24:38-46.

[3] LaPlante MP, Kaye HS. Demographics and Trends in Wheeled Mobility Equipment Use and Accessibility in the Community. Assist. Technol. 2010;22:317.

[4] Hoeman SP. Rehabilitation Nursing: Prevention, Intervention, and Outcomes. Elsevier Health Sciences; 2008.

[5] Curtis KA, Black K. Shoulder pain in female wheelchair basketball players. J. Orthop. Sports Phys. Ther. 1999;29:225-231.

[6] Finley MA, McQuade KJ, Rodgers MM. Scapular kinematics during transfers in manual wheelchair users with and without shoulder impingement. Clin. Biomech. 2005;20:32-40.

[7] Sonenblum SE, Sprigle SH. Some people move it, move it... for pressure injury prevention. J. Spinal Cord Med. 2016;0:1-5.

[8] Dalyan M, Cardenas DD, Gerard B. Upper extremity pain after spinal cord injury. Spinal Cord. 1999;37:191-195.

[9] Samuelsson K a. M, Tropp H, Gerdle B. Shoulder pain and its consequences in paraplegic spinal cord-injured, wheelchair users. Spinal Cord. 2004;42:41-46.

[10] Alm M, Saraste H, Norrbrink C. Shoulder Pain in Persons with Thoracic Spinal Cord Injury: Prevalence and Characteristics. J. Rehabil. Med. 2008;40:277-283.

[11] Bode RK, Heinemann AW, Kozlowski AJ, et al. Self-Scoring Templates for Motor and Cognitive Subscales of the FIM Instrument for Persons With Spinal Cord Injury. Arch. Phys. Med. Rehabil. 2014;95:676-679.e5.

[12] Janssen TWJ, van Oers CAJM, Veeger HEJ, et al. Relationship between physical strain during standardised ADL tasks and physical capacity in men with spinal cord injuries. Paraplegia. 1994;32:844-859.

[13] Toro ML, Koontz AM, Cooper RA. The Impact of Transfer Setup on the Performance of Independent Wheelchair Transfers. Hum. Factors J. Hum. Factors Ergon. Soc. 2012;0018720812460549.

[14] Koontz A, Toro M, Kankipati P, et al. An expert review of the scientific literature on independent wheelchair transfers. Disabil. Rehabil. Assist. Technol. $\underline{2012 ; 7: 20-29 .}$ 
[15] Martin J. Training and Performance Self-Efficacy, Affect, and Performance in Wheelchair Road Racers. Kinesiol. Health Sport Stud. [Internet]. 2002; Available from: http://digitalcommons.wayne.edu/coe khs/11.

[16] Best KL, Miller WC, Huston G, et al. Pilot Study of a Peer-Led Wheelchair Training Program to Improve Self-Efficacy Using a Manual Wheelchair: A Randomized Controlled Trial. Arch. Phys. Med. Rehabil. 2016;97:37-44.

[17] Sakakibara BM, Miller WC, Eng JJ, et al. Preliminary Examination of the Relation Between Participation and Confidence in Older Manual Wheelchair Users. Arch. Phys. Med. Rehabil. 2013;94:791-794.

[18] Gagnon D, Koontz A, Mulroy S, et al. Biomechanics of Sitting Pivot Transfers Among Individuals with a Spinal Cord Injury: A Review of the Current Knowledge. Top. Spinal Cord Inj. Rehabil. 2009;15:33-58.

[19] Barbareschi G, Cheng T-J, Holloway C. The effect of technique and transfer board use on the performance of wheelchair transfers. Healthc. Technol. Lett. [Internet]. 2017 [cited 2017 Oct 21]; Available from: http://digitallibrary.theiet.org/content/journals/10.1049/htl.2017.0075; jsessionid=6i0nui1279v 5g.X-iet-live-01.

[20] Haubert LL, Mulroy SJ, Hatchett PE, et al. Car Transfer and Wheelchair Loading Techniques in Independent Drivers with Paraplegia. Front. Bioeng. Biotechnol. [Internet]. 2015 [cited 2016 Feb 3];3. Available from: http://www.ncbi.nlm.nih.gov/pmc/articles/PMC4585017/.

[21] Crytzer TM, Cooper R, Jerome G, et al. Identifying research needs for wheelchair transfers in the built environment. Disabil. Rehabil. Assist. Technol. 2015;0:1-7.

[22] Hogaboom NS, Worobey LA, Boninger ML. Transfer Technique Is Associated With Shoulder Pain and Pathology in People With Spinal Cord Injury: A CrossSectional Investigation. Arch. Phys. Med. Rehabil. 2016;97:1770-1776.

[23] Deci EL, Ryan RM. Intrinsic motivation inventory. Self-Determ. Theory. 2003;

[24] Kelley K, Clark B, Brown V, et al. Good practice in the conduct and reporting of survey research. Int. J. Qual. Health Care. 2003;15:261-266.

[25] Desroches G, Gagnon D, Nadeau S, et al. Effects of sensorimotor trunk impairments on trunk and upper limb joint kinematics and kinetics during sitting pivot transfers in individuals with a spinal cord injury. Clin. Biomech. 2013;28:1-9.

[26] Nyland J, Quigley P, Huang C, et al. Preserving transfer independence among individuals with spinal cord injury. Spinal Cord Edegem. 2000;38:649-657.

[27] Taylor-Schroeder S, LaBarbera J, McDowell S, et al. The SCIRehab project: treatment time spent in SCI rehabilitation. Physical therapy treatment time during inpatient spinal cord injury rehabilitation., Physical therapy treatment time during inpatient spinal cord injury rehabilitation. J. Spinal Cord Med. J. Spinal Cord Med. 2011;34, 34:149, 149-161. 
[28] Ballinger DA, Rintala DH, Hart KA. The relation of shoulder pain and range-ofmotion problems to functional limitations, disability, and perceived health of men with spinal cord injury: a multifaceted longitudinal study. Arch. Phys. Med. Rehabil. 2000;81:1575-1581.

[29] McCasland LD, Budiman-Mak E, Weaver FM, et al. Shoulder Pain in the Traumatically Injured Spinal Cord Patient: Evaluation of Risk Factors and Function. JCR J. Clin. Rheumatol. 2006;12:179-186.

[30] Butler Forslund E, Roaldsen KS, Hultling C, et al. Concerns about falling in wheelchair users with spinal cord injury - validation of the Swedish version of the spinal cord injury falls concern scale. Spinal Cord. 2016;54:115-119.

[31] Jørstad EC, Hauer K, Becker C, et al. Measuring the Psychological Outcomes of Falling: A Systematic Review. J. Am. Geriatr. Soc. 2005;53:501-510.

[32] Ripat J, Woodgate R. The intersection of culture, disability and assistive technology. Disabil. Rehabil. Assist. Technol. 2011;6:87-96.

[1] Fliess-Dower O, Vanlandewijck YC, Van Der Woude LHV. Most Essential Wheeled Mobility Skills for Daily Life: An International Survey Among Paralympic Wheelchair Athletes With Spinal Cord Injury. Arch. Phys. Med. Rehabil. 2012:93:629-635.

[2] Mills T, Holm MB, Trefler E, et al. Development and consumer validation of the Functional Evaluation in a Wheelchair (FEW) instrument. Disabil. Rehabil. $2002 ; 24: 38-46$.

[3] LaPlante MP, Kaye HS. Demographics and Trends in Wheeled Mobility Equipment Use and Accessibility in the Commmnity. Assist. Technol. 2010;22:3 17.

[4] Hoeman SP. Rehabilitation Nursing: Prevention, Intervention, and Outcomes. Elsevier Health Sciences; 2008.

[5] Curtis KA, Black K. Shoulder pain in female wheelchair basketball players. J. Orthop. Sports Phys. Ther. 1999;29:225-231.

[6] Finley MA, McQuade KJ, Rodgers MM. Scapular kinematies during transfers in manual wheelchair users with and without shoulder impingement. Clin. Biomech. 2005;20:32 40 .

[7] Sonenblum SE, Sprigle SH. Some people move it, move it... for pressure injury prevention. J. Spinal Cord Med. 2016;0:1 5.

[8] Dalyan M, Cardenas DD, Gerard B. Upper extremity pain after spinal cord injury. Spinal Cord. 1999;37:191-195.

[9] Samuelsson K a. M, Tropp H, Gerdle B. Shoulder pain and its consequences in paraplegic spinal cord injured, wheelchair users. Spinal Cord. 2004;42:41 46. 
[10] Alm M, Saraste H, Norrbrink C. Shoulder Pain in Persons with Thoracic Spinal Cord Injury: Prevalence and Characteristics. J. Rehabil. Med. 2008;40:277 283.

[11] Bode RK, Heinemann AW, Kozlowski AJ, et al. Self Seoring Templates for Motor and Cognitive Subscales of the FIM Instrument for Persons With Spinat Cord Injury. Areh. Phys. Med. Rehabil. 2014;95:676-679.e5.

[12] Janssen TWJ, van Oers CAJM, Veeger HEJ, et al. Relationship between physical strain during standardised ADL tasks and physical capacity in men with spinal cord injuries. Paraplegia. 1994;32:844-859.

[13] Toro ML, Koontz AM, Cooper RA. The Impact of Transfer Setup on the Performance of Independent Wheelchair Transfers. Hum. Factors J. Hum. Factors Ergon. Soc. 2012;0018720812460549.

[14] Koontz A, Toro M, Kankipati P, et al. An expert review of the scientific literature on independent wheelchair transfers. Disabil. Rehabil. Assist. Technol. $2012 ; 7: 2029$.

[15] Martin J. Training and Performance Self-Efficacy, Affect, and Performance in Wheelchair Road Racers. Kinesiol. Health Sport Stud. [Internet]. 2002; Available from: http://digitalcommons.wayne.edu/coe_khs/11.

[16] Best KL, Miller WC, Huston G, et al. Pilot Study of a Peer Led Wheelchair Training Program to Improve Self Efficacy Using a Manual Wheelchair: A Randomized Controlled Trial. Arch. Phys. Med. Rehabil. 2016;97:37 44.

[17] Sakakibara BM, Miller WC, Eng JJ, et al. Preliminary Examination of the Relation Between Participation and Confidence in Older Manual Wheelchair Users. Areh. Phys. Med. Rehabil. 2013:94:791 794.

[18] Gagnen D, Koontz A, Mulroy S, et al. Biomechanies of Sitting Pivot Transfers Among Individuals with a Spinal Cord Injury: A Review of the Current Knowledge. Top. Spinal Cord Inj. Rehabil. 2009;15:33 58.

[19] Barbareschi G, Cheng T-J, Holloway C. The effect of technique and transfer board use on the performance of wheelchair transfers. Healthc. Technol. Lett. [Internet]. 2017 [ cited 2017 Oct 21]; Available from: http://digitallibrary.theiet.org/content/journals/10.1049/ht1.2017.0075; jsessionid=6i0nui1279v 5g.x-iet-live- 01 .

[20] Haubert LL, Mulroy SJ, Hatehett PE, et al. Car Transfer and Wheelchair Loading Techniques in Independent Drivers with Paraplegia. Front. Bioeng. Biotechnol. [Internet]. 2015 [cited 2016 Feb 3];3. Available from: http://www.ncbi.nlm.nih.gov/pme/articles/PMC4585017/.

[21] Crytzer TM, Cooper R, Jerome $G$, et al. Identifying research needs for wheelchair transfers in the built environment. Disabil. Rehabil. Assist. Technol. 2015;0:1 7.

[22] Hogaboom NS, Worobey LA, Boninger ML. Transfer Technique Is Associated With Shoulder Pain and Pathology in People With Spinal Cord Injury: A CrossSectional Investigation. Arch. Phys. Med. Rehabil. 2016;97:1770-1776. 
[23] Kelley K, Clark B, Brown V, et al. Good practice in the conduct and reporting of survey research. Int. J. Qual. Health Care. 2003;15:261 266.

[24] Deei EL, Ryan RM. Intrinsic motivation inventory. Self Determ. Theory. 2003;

[25] Desroches G, Gagnon D, Nadeau S, et al. Effects of sensorimotor trunk impairments on trunk and upper limb joint kinematics and kinetics during sitting pivot transfers in individuals with a spinal cord injury. Clin. Biomech. $2013 ; 28: 1-9$.

[26] Nyland J, Quigley P, Huang C, et al. Preserving transfer independence among individuals with spinal cord injury. Spinal Cord Edegem. 2000;38:649 657.

[27] Taylor-Schroeder S, LaBarbera J, McDowell S, et al. The SCIRehab project: treatment time spent in SCI rehabilitation. Physical therapy treatment time during inpatient spinal cord injury rehabilitation., Physical therapy treatment time during impatient spinal cord injury rehabilitation. J. Spinal Cord Med. J. Spinal Cord Med. 2011;34, 34:149, 149 161.

[28] Ballinger DA, Rintala DH, Hart KA. The relation of shoulder pain and range-ofmotion problems to functional limitations, disability, and perceived health of men with spinal cord injury: a multifaceted longitudinal study. Arch. Phys. Med. Rehabil. 2000;81:1575-1581.

[29] McCastand LD, Budiman-Mak E, Weaver FM, et al. Shoulder Pain in the Traumatically Injured Spinal Cord Patient: Evaluation of Risk Factors and Function. JCR J. Clin. Rheumatol. 2006;12:179 186.

[30] Butler Forslund E, Roaldsen KS, Hultling C, et al. Concerns about falling in wheelchair users with spinal cord injury validation of the Swedish version of the spinal cord injury falls concern seale. Spinal Cord. 2016;54:115 119.

[31] Jørstad EC, Hauer K, Becker C, et al. Measuring the Psychological Outcomes of Falling: A Systematic Review. J. Am. Geriatr. Soc. 2005;53:501 510.

[32] Ripat J, Woodgate R. The intersection of culture, disability and assistive technology. Disabil. Rehabil. Assist. Technol. 2011;6:87-96. 
Table 1 Demographic characteristics of participants

\begin{tabular}{|c|c|c|}
\hline Characteristic & Frequency & Percentage \\
\hline \multicolumn{3}{|l|}{ Gender } \\
\hline Male & 26 & $62 \%$ \\
\hline Female & 16 & $38 \%$ \\
\hline \multicolumn{3}{|l|}{ Age (years) } \\
\hline $18-25$ & 6 & $14.3 \%$ \\
\hline $26-35$ & 4 & $9.5 \%$ \\
\hline $36-45$ & 6 & $14.3 \%$ \\
\hline $46-55$ & 17 & $40.5 \%$ \\
\hline $56-65$ & 8 & $19.0 \%$ \\
\hline $65+$ & 1 & $2.4 \%$ \\
\hline \multicolumn{3}{|l|}{ Weight (kg) } \\
\hline less than $50 \mathrm{~kg}$ & 3 & $7.1 \%$ \\
\hline $50-70 \mathrm{~kg}$ & 17 & $40.5 \%$ \\
\hline $70-90 \mathrm{~kg}$ & 12 & $28.6 \%$ \\
\hline $90+\mathrm{kg}$ & 10 & $23.8 \%$ \\
\hline \multicolumn{3}{|l|}{ Height (cm) } \\
\hline less than $165 \mathrm{~cm}$ & 13 & $31 \%$ \\
\hline $165-180 \mathrm{~cm}$ & 16 & $38.1 \%$ \\
\hline $180-195 \mathrm{~cm}$ & 12 & $28.6 \%$ \\
\hline $195+\mathrm{cm}$ & 1 & $2.4 \%$ \\
\hline \multicolumn{3}{|c|}{ Experience as a wheelchair user } \\
\hline Less than 1 year & 2 & $4.8 \%$ \\
\hline $1-5$ & 10 & $23.8 \%$ \\
\hline $6-10$ & $8=$ & $16.7 \%$ \\
\hline $10+$ & +2 & $54.8 \%$ \\
\hline \multicolumn{3}{|c|}{ Type of primary wheelchair } \\
\hline MW rigid frame & 19 & $45.2 \%$ \\
\hline MW foldable frame & 10 & $23.8 \%$ \\
\hline Powered wheelchair & 11 & $26.2 \%$ \\
\hline PAPAW & 2 & $4.8 \%$ \\
\hline \multicolumn{3}{|l|}{ Medical condition } \\
\hline SCI (paraplegia) & 15 & $35.7 \%$ \\
\hline SCI (quadriplegia) & 7 & $16.7 \%$ \\
\hline MS & 2 & $4.8 \%$ \\
\hline $\mathrm{CP}$ & 3 & $7.1 \%$ \\
\hline Other & 15 & $35.7 \%$ \\
\hline
\end{tabular}




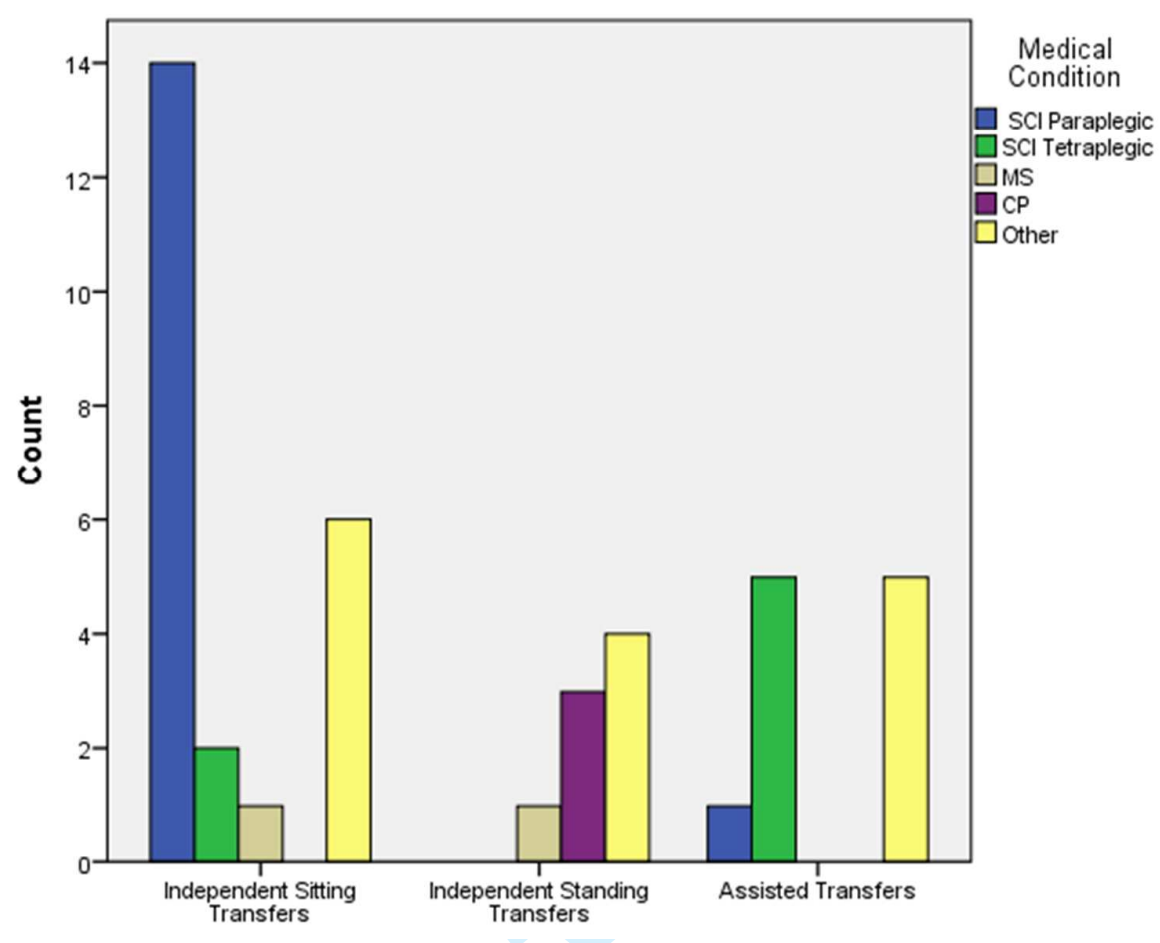

Figure 1 Bar chart showing distribution of participants' medical condition according to transferring technique 


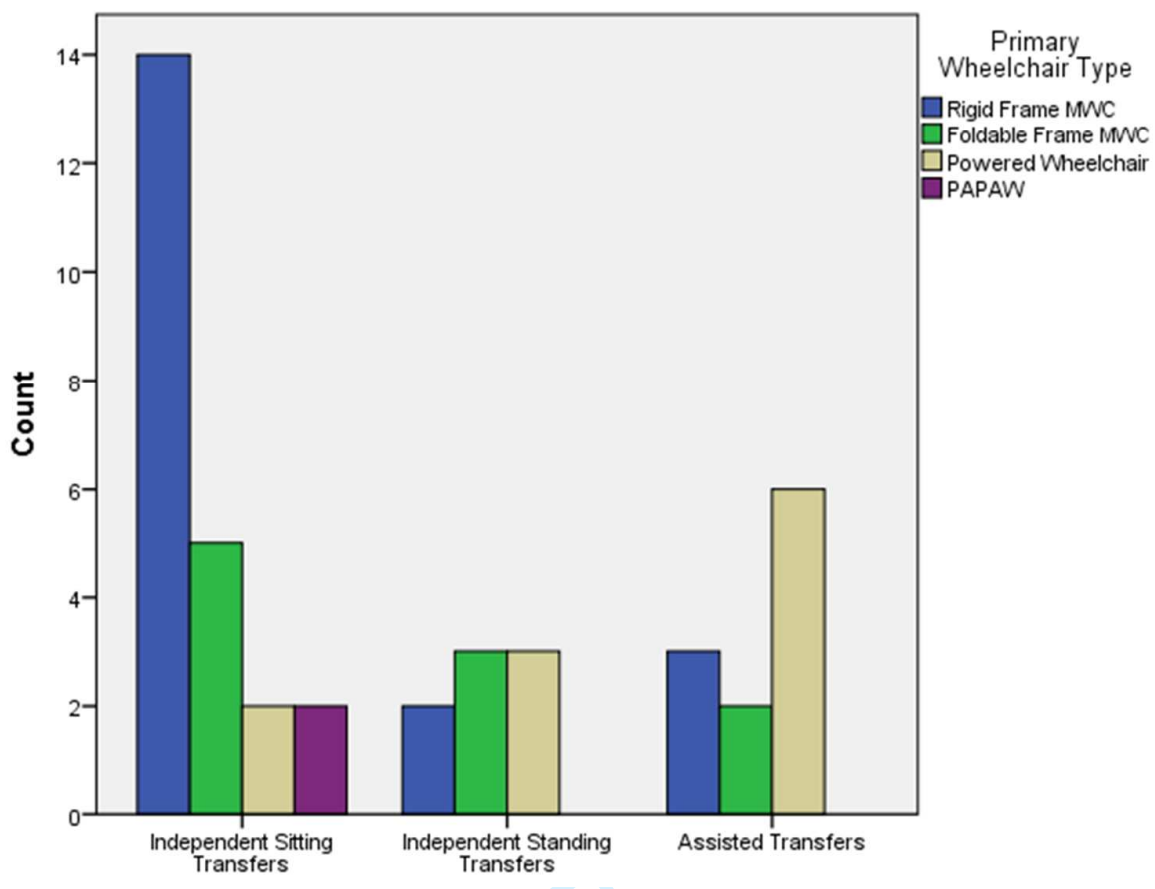

Figure 1 Bar chart showing distribution of participants' primary wheelchair type according to transferring technique 


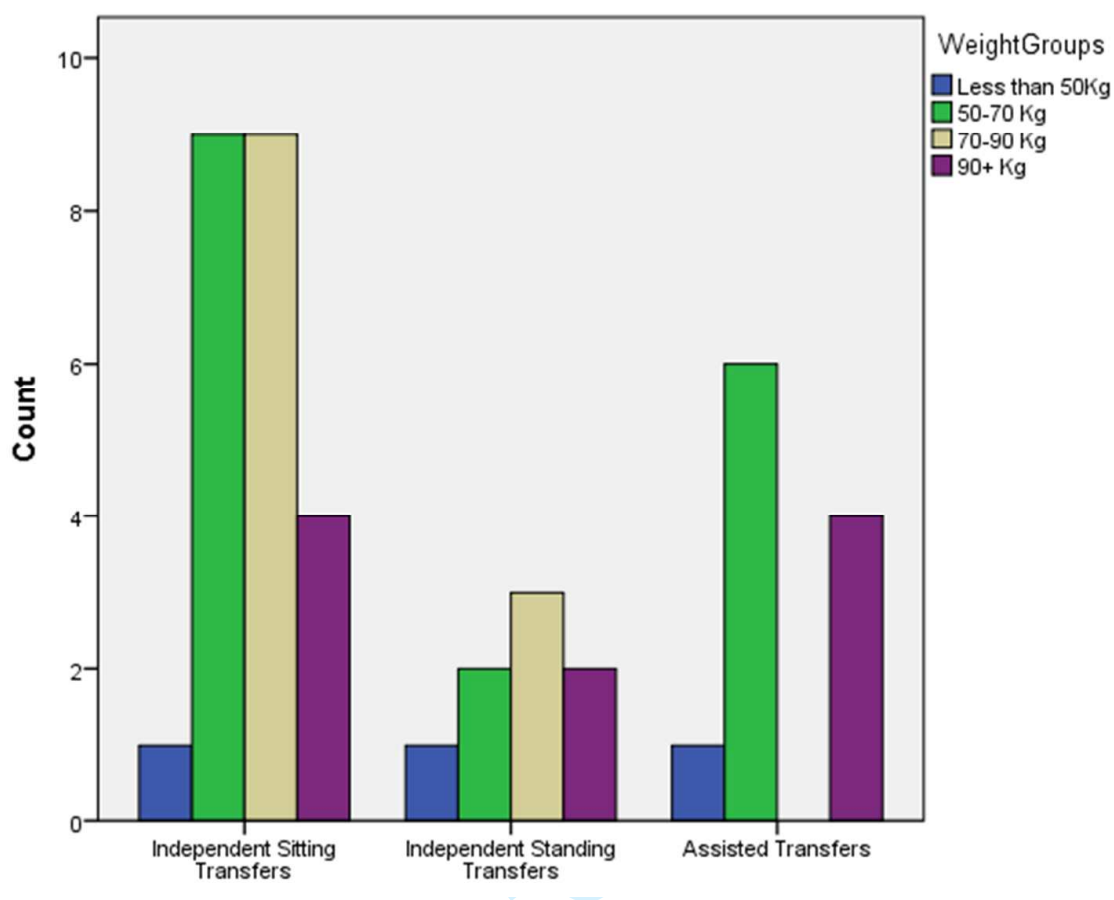

Figure 1 Bar chart showing distribution of participants' weight according to transferring technique 


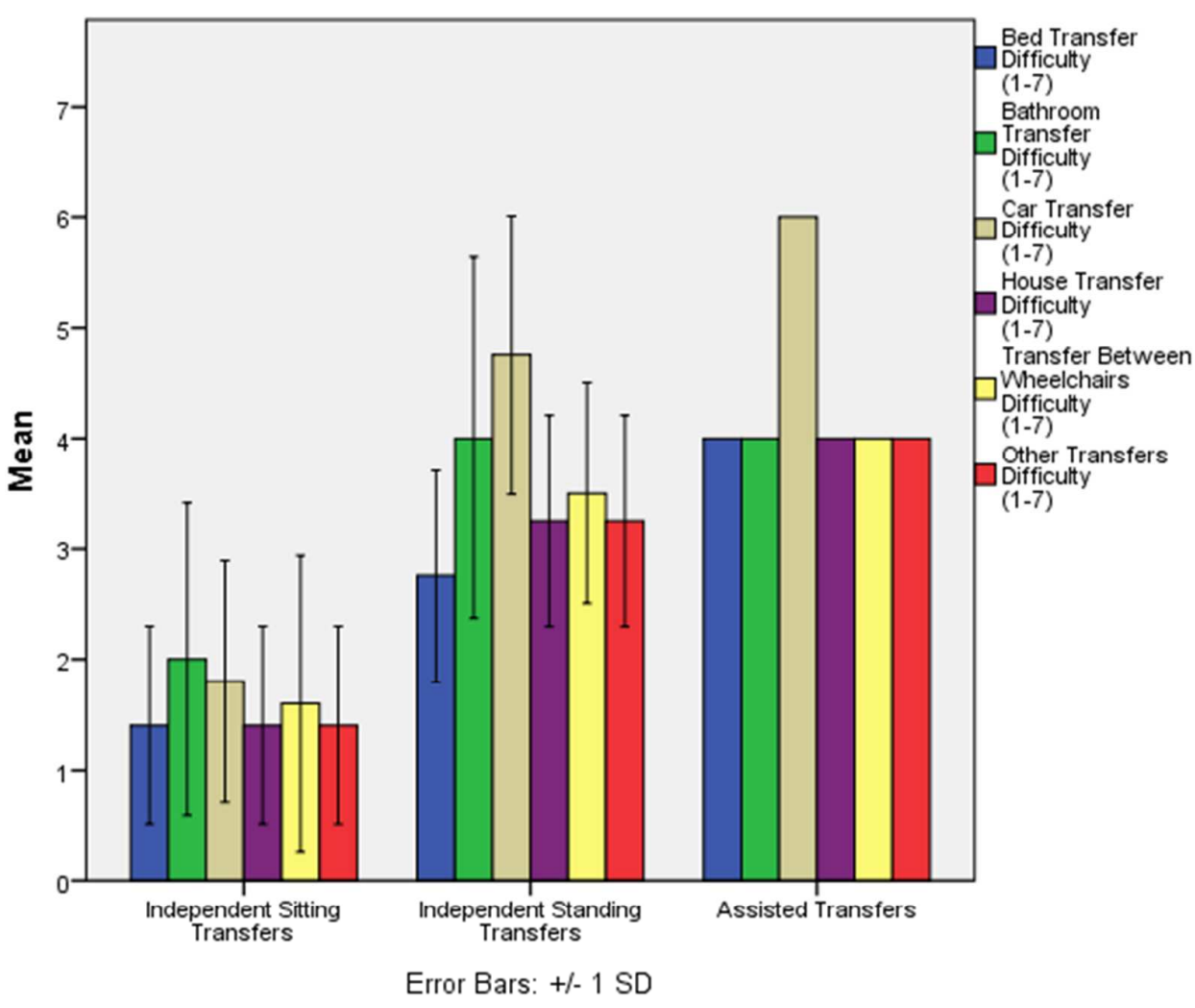

Figure 1 Bar chart showing mean reported difficulty for different types of transfers according to individuals' transferring technique 


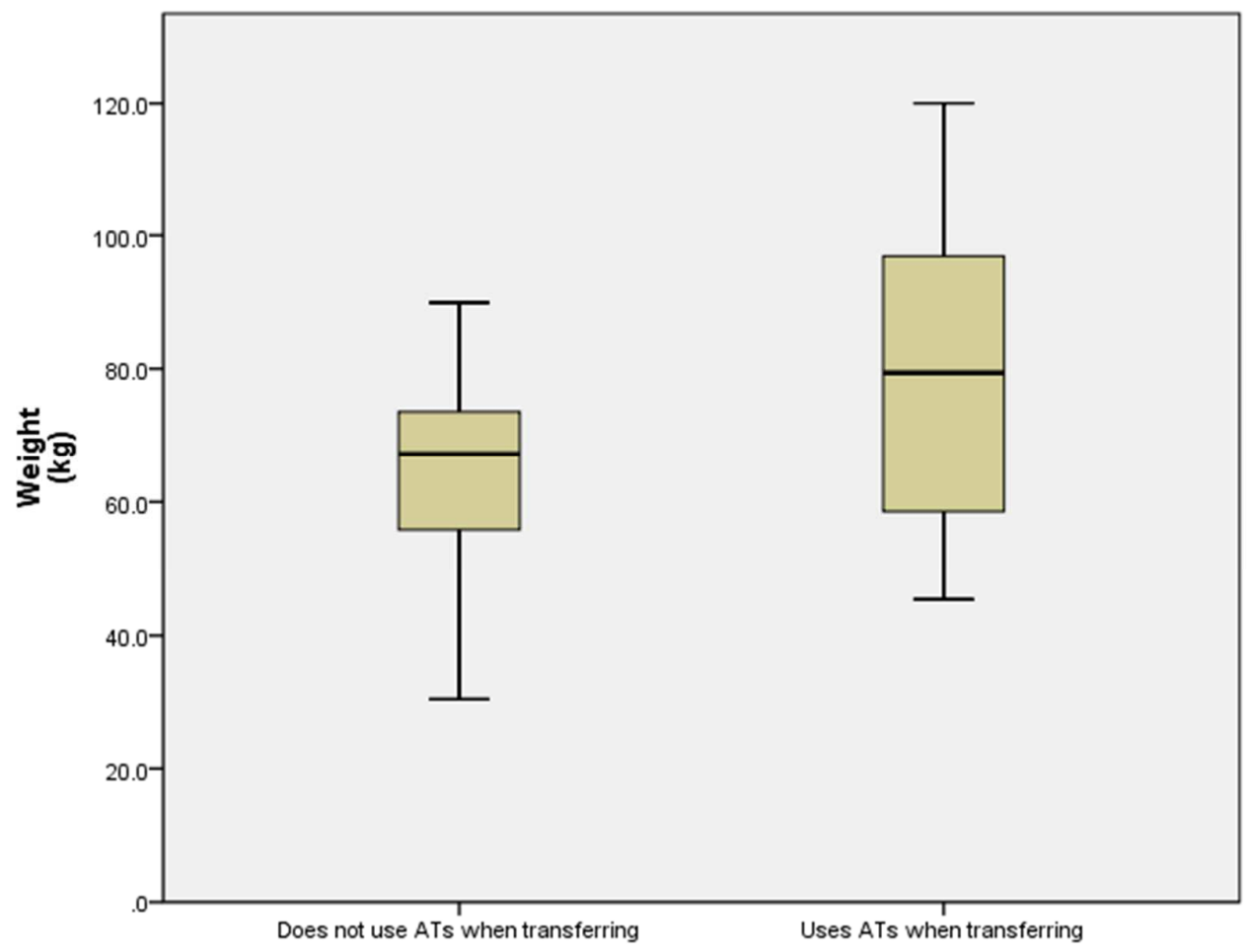

Figure 1 Boxplot showing weight differences between participants who use (or do not use) ATs when transferring 
2

3

4

5

6

7

8

9

10

11

12

13

14

15

16

17

18

19

20

21

22

23

24

25

26

27

28

29

30

31

32

33

34

35

36

37

38

39

40

41

42

43

44

45

46

47

48

49

50

51

52

53

54

55

56

57

58

59

60
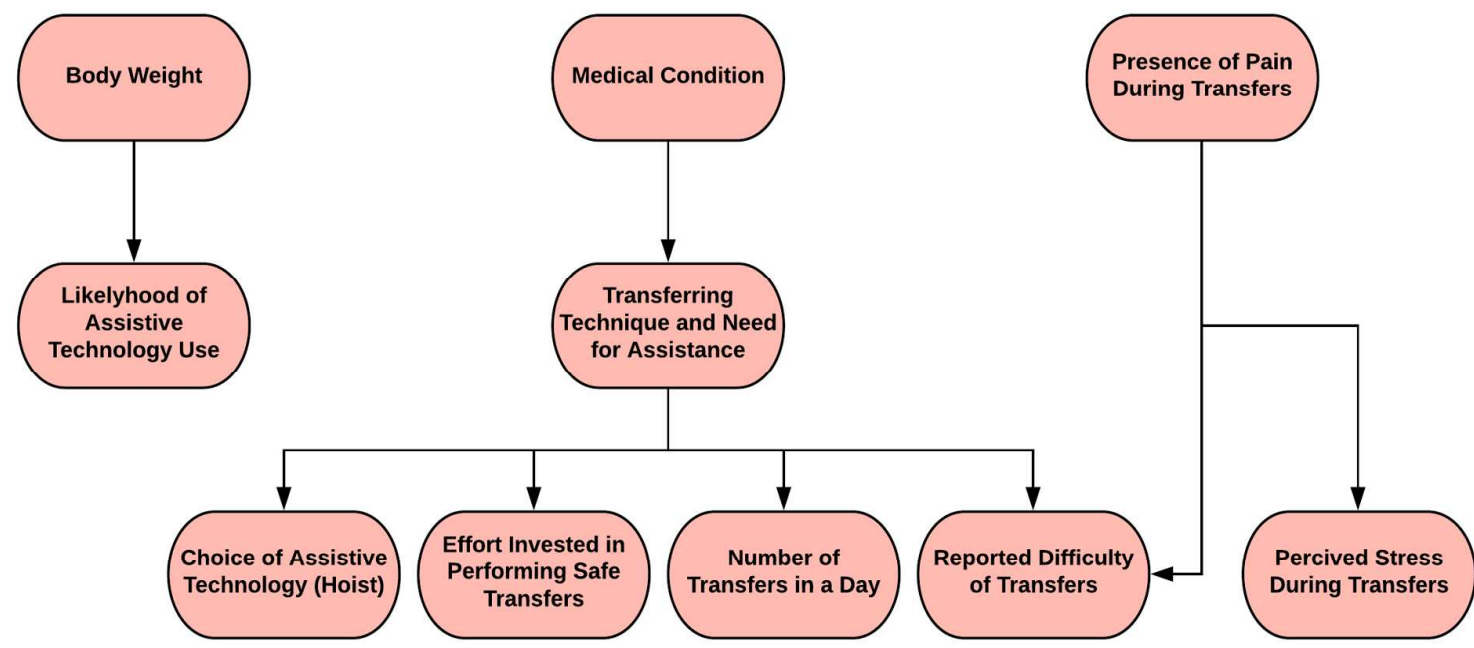

Figure 6 Diagram illustrating all the significant relationships, identified in the current survey, between the various factors related to the performance of wheelchair transfers. 


\section{Implications for Rehabilitation}

- Transferring technique is usually determined by the individual's impairment. In turn, transferring technique will greatly affect the perceived difficulty of different types of wheelchair transfers and, the number of transfers that people will perform in a day.

- The performance of wheelchair transfers exacerbate the painful symptomatology of the person, regardless of their medical condition or transferring technique. The presence of pain increases the reported difficulty of wheelchair trasnfers.

- Use of assistive technologies during transfers is linked to the increased body weight of the person, while the choice of the type of assistive technology seems to be dependent of the transferring technique used by the person. Overall, available ATs are only moderately effective in enabling people to perform wheelchair transfer and they can be cause of frustration for the users due to their high cost and limited functionality. 